\title{
AN ACCURATE PSF MODEL WITH FEW PARAMETERS FOR AXIALLY SHIFT-VARIANT DECONVOLUTION
}

\author{
François Aguet, Dimitri Van De Ville, and Michael Unser \\ Biomedical Imaging Group \\ Ecole Polytechnique Fédérale de Lausanne
}

\begin{abstract}
Accurate knowledge of an imaging system's point spread function (PSF) is crucial for successful deconvolution. For fluorescence microscopy, PSF estimations based on either theoretical models or experimental measurements are available. However, due to the axially shift-variant nature of the PSF, neither method guarantees an estimate that is valid for the entire object space. In this work, we present a reducedparameter version of a state-of-the-art theoretical model. We give a maximum-likelihood based algorithm for the estimation of its parameters, and we show how a fit of our model to two axially isolated point source measurements in an experimental setup can be used to accurately reproduce measured PSFs within the entire specimen.
\end{abstract}

Index Terms - Deconvolution, Fluorescence microscopy, Maximum-likelihood, Point spread function

\section{INTRODUCTION}

For the deconvolution of microscopy data, precise knowledge of the acquisition system's point spread function (PSF) is highly desirable, due to its critical influence on the quality of the restoration results [1]. In order to obtain a suitable estimate, the PSF can be modeled theoretically, by taking into account the system's optical parameters and properties, or measured experimentally, by recording the 3-D image of sub-resolution point sources. The principal difficulty in obtaining a representative model resides in the strong axial shift-variance observed under most experimental conditions [2]. This originates from the mismatch that generally occurs between the refractive indices of the immersion medium and the specimen, which results in increasingly strong aberrations as the point source moves deeper into the specimen [3].

A theoretical approach has the advantage of generating a diffraction-limited model that can be evaluated for the entire object space, but, due to the difficulty of accurately determining a modern system's parameters, the result is not guaranteed

This work was supported by the Swiss National Science Foundation under grant 200020-109415, as well as by the Hasler foundation under grant 2033. to match experimental conditions. Conversely, an experimentally measured PSF is a locally accurate representation of the system's properties, but is usually not globally valid due to the axial shift-variance. Furthermore, the procedure often gets tedious, as several measurements need to be averaged due to noise degradation and background fluorescence.

In this paper, we propose a combination of both approaches that aims at providing a theoretical representation of actual experimental conditions. This is achieved by fitting a parametric theoretical model to experimental measurements. However, most experimentally validated PSF models depend on a large number of parameters, which renders them unsuitable for a global optimization approach. We derive a reduced-parameter version of the scalar model proposed by Gibson and Lanni [3]. We also present a framework of image formation that takes into account various sources of noise, as well as detector pixelation. Subsequently, we describe the maximum-likelihood (ML) estimation for the relevant parameters, and show that our model correctly predicts measurements in an experiment where fluorescent beads are embedded at different depths. Fitted to two measurements at the axial extremes of the observed object space, we evaluate our model for intermediate axial positions and compare the result with the corresponding bead measurements.

\section{IMAGE FORMATION MODEL}

State-of-the-art scalar [3] and vectorial [4] models describe phase aberrations based on the computation of the optical path difference (OPD) between design conditions and experimental conditions in the sample setup [5]. These optical paths are calculated over the three layers that are present in every sample setup: the immersion layer, the coverslip, and the specimen layer, with reference and experimental values for the thickness and refractive index of each. This gives rise to a number of unknown parameters that renders a global fit of the model to an experimental measurement ill-posed; i.e., different combinations of parameter values can result in the same or in a highly similar phase aberration. Furthermore, index mismatches originating from these three layers are not the only possible sources of aberration. Many high-NA objectives are equipped with a corrective ring designed to compensate for 
aberrations induced by a mismatched coverslip thickness. As the calibration of this ring is performed empirically, a rigorous model would require an extension rather than a simplification of the OPD. In order to achieve a sufficient degree of simplicity, we introduce a model based on two layers only: the standard specimen layer, and a layer combining all others. As we will see, the mismatch of refractive indices between these two layers is sufficient for representing mismatch-induced aberrations in the PSF. Note that this model is still exact when the immersion medium and coverslip conform to design conditions.

We start by introducing the scalar PSF that our model is based on, and give the corresponding OPD term. Subsequently, we formulate a general model for acquisition noise, and briefly discuss the effects of discretization and pixelation by the CCD array.

\subsection{PSF model}

Phase aberrations are most accurately characterized by the vectorial models proposed by Hell et al. [4] and Török et al. [6]. These models are computationally costly, however, and offer no significant gain in accuracy for the application discussed here with respect to the scalar one proposed by Gibson and Lanni [3]. The latter model takes the general form

$$
h\left(\mathbf{x}, \mathbf{x}_{p} ; \mathbf{p}\right)=\left|A \int_{0}^{1} e^{i W\left(\rho, \mathbf{x}, \mathbf{x}_{p} ; \mathbf{p}\right)} J_{0}(k r \mathrm{NA} \rho) \rho \mathrm{d} \rho\right|^{2},
$$

where $\mathbf{x}=(x, y, z)$ is a point on the image plane in object space, $\mathbf{x}_{p}=\left(x_{p}, y_{p}, z_{p}\right)$ are the coordinates of the point source, $\mathbf{p}=(\mathrm{NA}, \mathbf{n}, \mathbf{t})$ is a parameter vector containing the optical parameters of the system: numerical aperture (NA), refractive index (n) and thickness (t) of the individual layers, $r=\sqrt{\left(x-x_{p}\right)^{2}+\left(y-y_{p}\right)^{2}}, A$ is a constant amplitude, and $J_{0}$ denotes the Bessel function of the first kind of order zero. For ease of notation, all coordinates are expressed in object space, which implies that we consider the image space to be demagnified and projected into object space. The phase term $W\left(\rho, \mathbf{x}, \mathbf{x}_{p} ; \mathbf{p}\right)=k_{0} \Lambda\left(\rho, \mathbf{x}, \mathbf{x}_{p} ; \mathbf{p}\right)$, where $k_{0}$ is the vacuum wave number, is defined by the optical path difference $\Lambda\left(\rho, \mathbf{x}, \mathbf{x}_{p} ; \mathbf{p}\right)$ between the actual and ideal imaging conditions (further details are given in $[3,7]$ ).

\subsection{Reduced-parameter OPD model}

For a simplified model consisting of two layers (see Fig. 1), we define the OPD as

$$
\begin{array}{r}
\Lambda\left(\rho, \mathbf{x}, \mathbf{x}_{p} ; \mathbf{p}\right)=z_{p} \sqrt{n_{s}^{2}-\mathrm{NA}^{2} \rho^{2}}-t_{c}^{*} \sqrt{n_{c^{*}}^{2}-\mathrm{NA}^{2} \rho^{2}} \\
\quad+\left(z_{p}-z_{n}+n_{c}\left(-\frac{z_{p}}{n_{s}}+\frac{t_{c}^{*}}{n_{c}^{*}}\right)\right) \sqrt{n_{c}^{2}-\mathrm{NA}^{2} \rho^{2}}
\end{array}
$$

where $z_{n}$ denotes the position of the focal plane, and $n_{c}$ and $n_{s}$ denote the refractive indices of the combined layers and

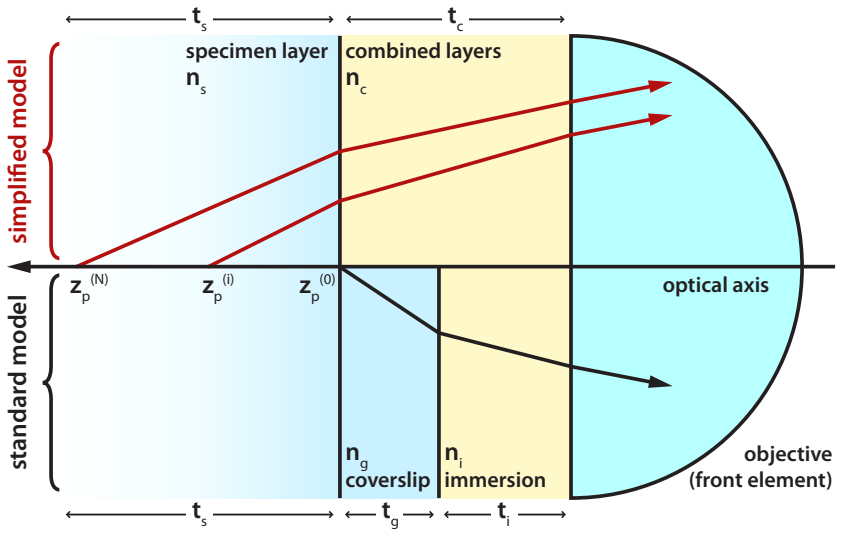

Fig. 1. Schematic representation of the optical path difference. Design conditions are satisfied when the source is located at the specimen/coverslip interface, and no index mismatches occur. The red rays illustrate deviations from these conditions for sources located at arbitrary depths.

specimen layer, respectively. The parameters $n_{c}^{*}$ and $t_{c}^{*}$ are the design values for the refractive index and thickness of the combined layers. In the Gibson and Lanni model, mismatched values for the coverslip and immersion medium contribute to the phase aberration individually. In practice, however, a corrective ring on the objective serves to minimize the contribution of coverslip-induced aberrations, which justifies our proposed approximation. Consequently, the appropriate reference values for this layer are given as the working distance of the objective for $t_{c}^{*}$, and the design refractive index of the immersion medium for $n_{c}^{*}$.

\subsection{Noise model}

The dominant sources of noise in fluorescence microscopy are shot noise, background noise due to autofluorescence of the sample, and read-out noise. Whereas the first two obey Poisson statistics, the latter is Gaussian distributed, which calls for an additive noise model. The Poisson distribution rapidly converges towards a Gaussian one with equal mean and variance, given that the variance is large enough (this is usually considered the case when $\sigma^{2}>10$, and is a valid assumption for the read-out noise observed in our measurements). Taking this into account, we establish a general noise model where we formulate the expected photon count $\bar{q}\left(\mathbf{x}, \mathbf{x}_{p} ; \mathbf{p}\right)$ corresponding to a point $\mathbf{x}$ in object space as

$$
\bar{q}\left(\mathbf{x}, \mathbf{x}_{p} ; \mathbf{p}\right)=c \cdot\left(h\left(\mathbf{x}, \mathbf{x}_{p} ; \mathbf{p}\right)+\sigma_{b}^{2}\right)
$$

where $c$ is a conversion factor and $\sigma_{b}^{2}$ is the variance (in intensity) of the read-out noise. To simplify the notation, we will omit the argument $\left(\mathbf{x}, \mathbf{x}_{p} ; \mathbf{p}\right)$ when it is clear from the context. The probability of detecting $q$ photons at $\mathbf{x}$ is then given 
by

$$
P\left(q\left(\mathbf{x}, \mathbf{x}_{p} ; \mathbf{p}\right)\right)=\frac{e^{-\bar{q}}(\bar{q})^{q}}{q !} .
$$

\subsection{Pixelation}

The pixelation of the detector has a non-negligible effect on the measured intensity distribution of a point source, and must therefore be taken into account by the model. Hereinafter, we assume that a point of observation $\mathbf{x}$ represents a pixel on the $\mathrm{CCD}$, and thus that, when appropriate, functions of $\mathrm{x}$ incorporate integration over the pixel's area (pixels are assumed to be contiguous and non-overlapping).

\section{ESTIMATION ALGORITHM}

A fit of the proposed model to experimental data requires the estimation of the following parameters: the positions $z_{n}$ of the acquisitions, the position $z_{p}$ of the point source, and the refractive indices $n_{c}$ and $n_{s}$. Performing a global fit for all parameters would still involve a large search space. Therefore, our algorithm relies on a specific experimental setup; i.e., it requires at least one point source to be located at the specimen/coverslip interface, which is straightforward to achieve in practice (details are given in Section 4.1). For a source located at this interface, the only remaining parameters in the OPD are $z_{n}$ and $n_{c}$ (since $z_{p}=0$ ). Furthermore, in the presence of aberrations, the intensity maximum of the PSF is shifted with respect to the position of the source. Although this shift is nonlinear and cannot be expressed in closed form, it can be exploited to estimate the positions $z_{n}$ of the acquisitions, assuming that the other parameters are known.

A reliable fit is obtained by performing the following estimation procedure:

1. ML estimation of $n_{c}$, using the intensity-shift property to interpolate for $z_{n}$.

2. ML estimation for the fine-scale adjustment of $z_{n}$. This yields a global reference for all acquisition positions in the experiment.

Values for the remaining two parameters are estimated in similar fashion; instead of a point source at the specimen/coverslip interface, a source at the farthest relevant point in the sample is selected. The intensity shift is exploited during the ML estimation of the refractive index $n_{s}$, after which the position $\mathbf{x}_{p}$ is localized to higher accuracy using the appropriate ML estimator.

\subsection{Maximum-likelihood estimators}

We now provide a general description of the ML estimators used by our algorithm. The joint probability of observing a given spatial distribution $q\left(\mathbf{x}, \mathbf{x}_{p} ; \mathbf{p}\right)$ of photons emitted by a source located at $\mathbf{x}_{p}$ is given by

$$
\prod_{\mathbf{x} \in \mathcal{S}} P\left(q\left(\mathbf{x}, \mathbf{x}_{p} ; \mathbf{p}\right)\right),
$$

where $\mathcal{S}$ is the set of voxels in the acquisition. By maximizing the likelihood of Eq. (5) with respect to a parameter $\theta$, we obtain the basis for our estimators:

$$
\frac{\partial}{\partial \theta} \ln \prod_{\mathbf{x} \in \mathcal{S}} P(q)=\sum_{\mathbf{x} \in \mathcal{S}} \frac{\partial \bar{q}}{\partial \theta}\left(\frac{q}{\bar{q}}-1\right) \equiv 0 .
$$

Since there is no closed-form solution for $\theta$ in the above expression, we take the first-order Taylor approximation of the maximum-likelihood function around an estimate $\hat{\theta}$ of $\theta$, which gives us the following iterative expression:

$$
\theta^{(m+1)}=\theta^{(m)}-\frac{\sum_{\mathbf{x} \in \mathcal{S}}\left(\frac{\partial \bar{q}}{\partial \theta}\left(\frac{q}{\bar{q}}-1\right)\right)}{\sum_{\mathbf{x} \in \mathcal{S}}\left(\frac{\partial^{2} \bar{q}}{\partial \theta^{2}}\left(\frac{q}{\bar{q}}-1\right)-\left(\frac{\partial \bar{q}}{\partial \theta}\right)^{2} \frac{q}{(\bar{q})^{2}}\right)},
$$

where $m$ denotes the iteration.

\section{RESULTS}

We first evaluated the performance of the proposed model and algorithm for synthetic data, which was generated based on the complete Gibson and Lanni model. Parameter values were retrieved without any significant loss in accuracy as compared to a fitting approach that operates on all acquisitions simultaneously.

\subsection{Experimental setup}

For the experimental validation of our model, we prepared samples consisting of fluorescent beads (170 nm PS-Speck microspheres from Invitrogen) distributed over a thick layer ( $>20 \mu \mathrm{m}$ ) of mounting medium. A diluted solution of beads was dried onto a coverslip and embedded in a solid mounting medium (Dako GlycerGel). The sample was then imaged using a $63 \times, 1.4$ NA oil immersion objective from Leica (HCX PL APO CS). Following standard experimental procedure, the correction ring on the objective was calibrated on a Leica TCS-SP2 AOBS confocal microscope, in order to minimize coverslip-induced aberrations.

The results from fitting our model to these measurements are shown in Fig. 2. In Fig. 2-(a), an xz-section of a bead located at the coverslip/specimen interface is shown (note its slight asymmetry); an analogous section of a source located at the other extremity of the acquisition volume is shown in Fig. 2-(e). The output of the fitting algorithm is shown under (b) and (f), respectively. Using the resulting parameterization 

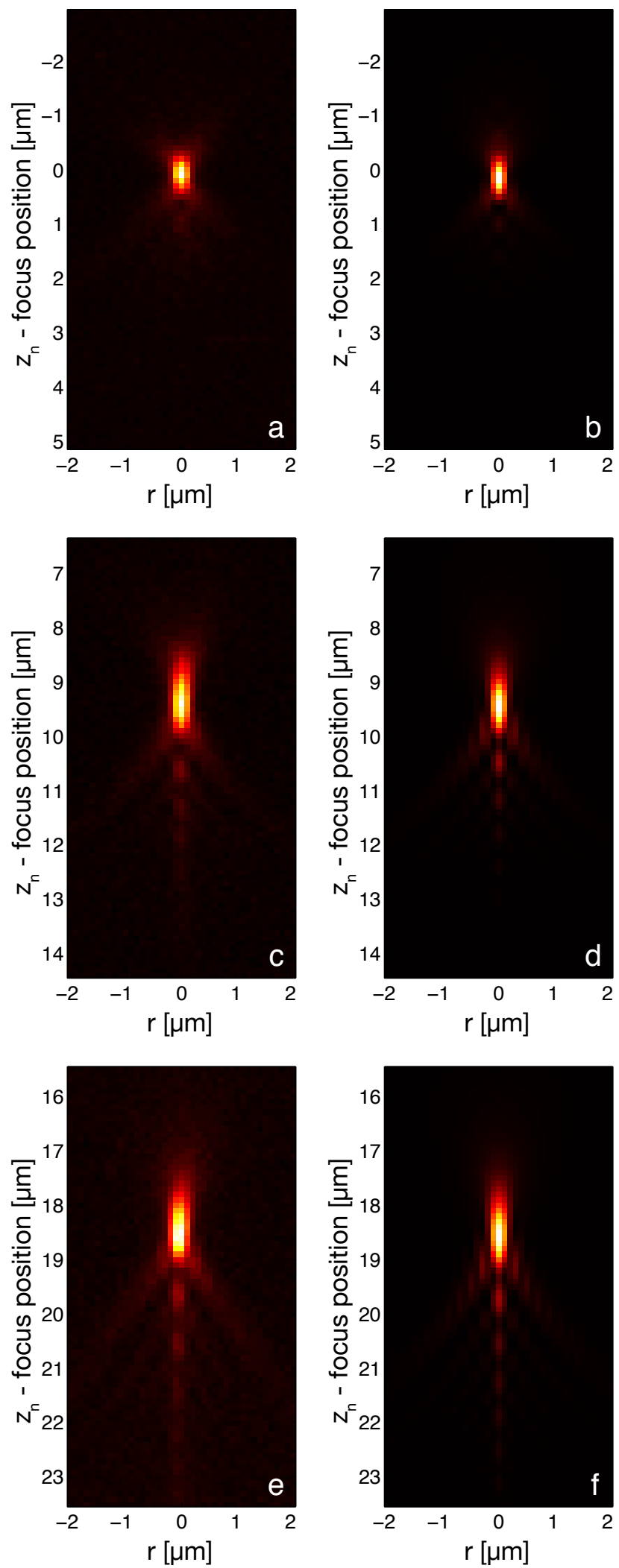

Fig. 2. Fit of the proposed model (b, d, f) to a set of experimental measurements (a, c, e). Details are given in the text, in Section 4.1 . of the model, the position $\mathbf{x}_{p}$ of the intermediate sources can then be localized; an example of an acquisition is shown in Fig. 2-(c), with the corresponding localized fit in Fig. 2-(d). An optimal correspondence between the model and experimental data is obtained.

\subsection{Discussion}

The quality of the fitted model for experimental data is highly promising for the application of shift-variant deconvolution [8]. Calibrating the model by means of isolated point sources in the sample is feasible to implement in practice; a similar sample preparation to the one described can be used to include fluorescent beads in a biological specimen. The results presented in this paper are also relevant for shift-invariant deconvolution, where a model that matches experimental conditions and correctly reproduces the asymmetry of the PSF for a source at a given depth within the specimen can lead to noticeably improved deconvolution results [2].

The proposed approach is also computationally efficient; in our implementation, the evaluation of a PSF on $10^{6}$ points, together with the relevant derivatives, is achieved in less than 1.3 seconds (on a $2.66 \mathrm{Ghz}$ Intel Xeon CPU).

Future work includes validation of the model on more complex, biological samples, where the refractive index in the specimen can undergo local variations, as well as the application of our model to shift-variant deconvolution.

\section{ACKNOWLEDGMENTS}

The authors would like to thank Boris Zarda (Leica Microsystems) for his help with the preparation and imaging of the test samples.

\section{REFERENCES}

[1] J. G. McNally, T. Karpova, J. Cooper, and J. A. Conchello, "Threedimensional imaging by deconvolution microscopy," Methods, vol. 19, pp. 373-385, 1999.

[2] B. A. Scalettar, J. R. Swedlow, J. W. Sedat, and D. A. Agard, "Dispersion, aberration and deconvolution in multi-wavelength fluorescence images," J. Microsc., vol. 182, no. 1, pp. 50-60, 1996.

[3] S. F. Gibson and F. Lanni, "Experimental test of an analytical model of aberration in an oil-immersion objective lens used in three-dimensional light microscopy," J. Opt. Soc. Am. A, vol. 8, no. 10, pp. 1601-1613, 1991.

[4] S. Hell, G. Reiner, C. Cremer, and E. H. K. Stelzer, "Aberrations in confocal fluorescence microscopy induced by mismatches in refractive index," J. Microsc., vol. 169, no. 3, pp. 391-405, 1993.

[5] O. Haeberlé, "Focusing of light through a stratified medium: a practical approach for computing microscope point spread functions. Part I: Conventional microscopy," Opt. Commun., vol. 216, pp. 55-63, 2003.

[6] P. Török and P. Varga, "Electromagnetic diffraction of light focused through a stratified medium," Appl. Opt., vol. 36, no. 11, pp. 2305-2312, 1997.

[7] F. Aguet, D. Van De Ville, and M. Unser, "A maximum-likelihood formalism for sub-resolution axial localization of fluorescent nanoparticles," Opt. Express, vol. 13, no. 26, pp. 10503-10522, 2005.

[8] C. Preza and J. A. Conchello, "Depth-variant maximum-likelihood restoration for three-dimensional fluorescence microscopy," J. Opt. Soc. Am. A, vol. 21, no. 9, pp. 1593-1601, 2004. 